Ryner Jose D. Carrillo, MD, MSc1,2

Precious Eunice R. Grullo, MD, MPH

Maria Luz M. San Agustin, RN, MClinAudio ${ }^{1,3}$

'Department of Otorhinolaryngology

Philippine General Hospital, University of the Philippines

Manila

2Department of Anatomy

College of Medicine, University of the Philippines Manila

${ }^{3}$ Philippine National Ear Institute

National Institutes of Health, University of the Philippines Manila

\section{A Cotton Wick Improves Hearing in a Patient with Profound Hearing Loss}

Dear Editor,

The tympanic membrane and the ossicular chain contribute roughly $28 \mathrm{~dB}$ in hearing gain. In chronic suppurative otitis media, loss of tympanic membrane and lysis of the ossicular chain are significant causes of hearing loss. ${ }^{1}$ Through the years, hearing impairment has been augmented using various devices such as ear trumpets, carbon hearing aids, vacuum tube and transistor hearing aids, bone anchored hearing aids and cochlear implants. ${ }^{2}$ This case report describes how a cotton wick was used to amplify sound.

\section{CASE REPORT}

A 65-year-old man consulted for hearing loss. He had a childhood history of recurrent ear discharge and hearing loss and was diagnosed with chronic suppurative otitis media. At age 55, he underwent tympanomastoidectomy of the left ear. While surgery stopped the left ear discharge, there was complete hearing loss in this ear. For this reason, he opted not to have surgery on the right ear. There was subsequent recurrent ear disease of the right ear. He would clean his ear with a cotton wick and apply antibiotic drops during bouts of ear discharge. He observed that leaving the ear wick with a few drops of topical otic preparations (polymyxin-neomycin-steroid or ofloxacin) would lessen the frequency of ear discharge and improve his hearing. He found that morning application and positioning of the cotton wick in his right ear using tweezers and a toothpick allowed him to hear adequately to conduct his daily activities as an architect. (Figure 1, 2) The fear of hearing loss from another surgery, cost of a commercial hearing aid and great utility of a simple cotton wick made him continue his practice for these ten years.

Examination of the right middle ear without the cotton wick showed thickened mucosa, absent malleus and incus structures, a patent Eustachian tube and a near - total tympanic membrane perforation. There was no keratinous material or foul smelling discharge. (Figure 3) Pure tone audiometry confirmed that with the cotton wick, the right air-bone gap decreased at $500 \mathrm{hz}, 1 \mathrm{kHz}, 2 \mathrm{Kh}$ and $4 \mathrm{KHz}$ by $30 \mathrm{db}, 40 \mathrm{~dB}, 35 \mathrm{~dB}$ and $25 \mathrm{~dB}$, respectively. (Table 1) 


\section{LETTER TO THE EDITOR}

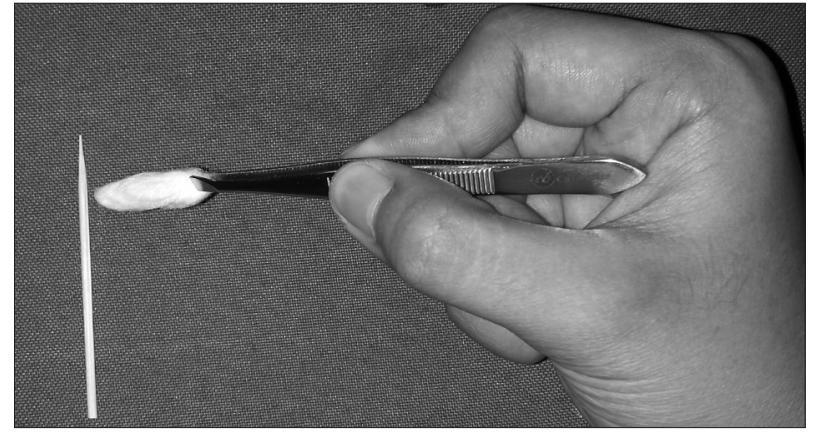

1. Cotton wick is shaped using a toothpick and tweezers applied in the ear canal and repositioned using the same tools while performing a valsalva maneuver.

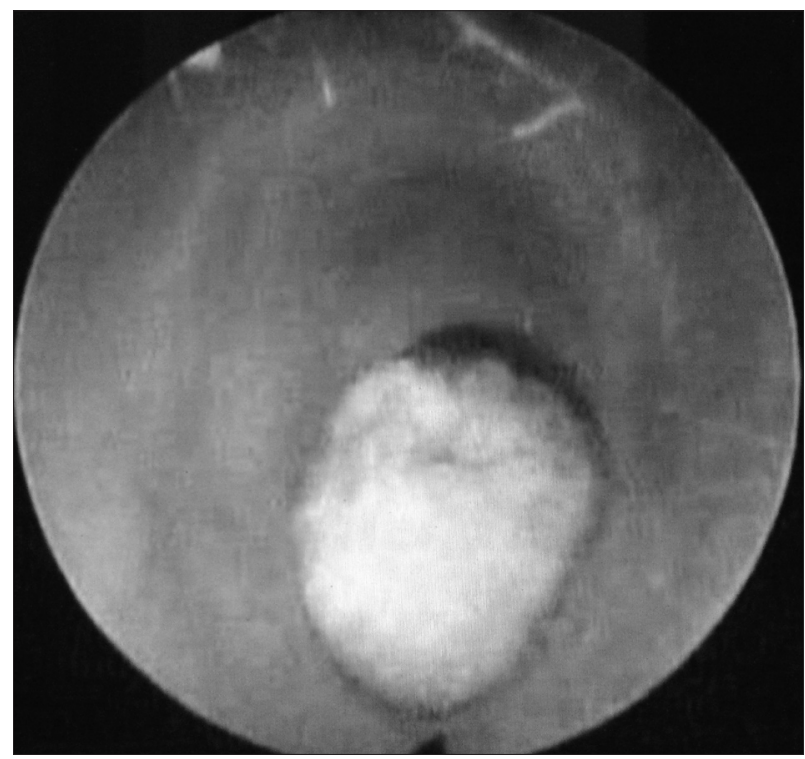

Figure 2. Cotton wick positioned in right ear canal.

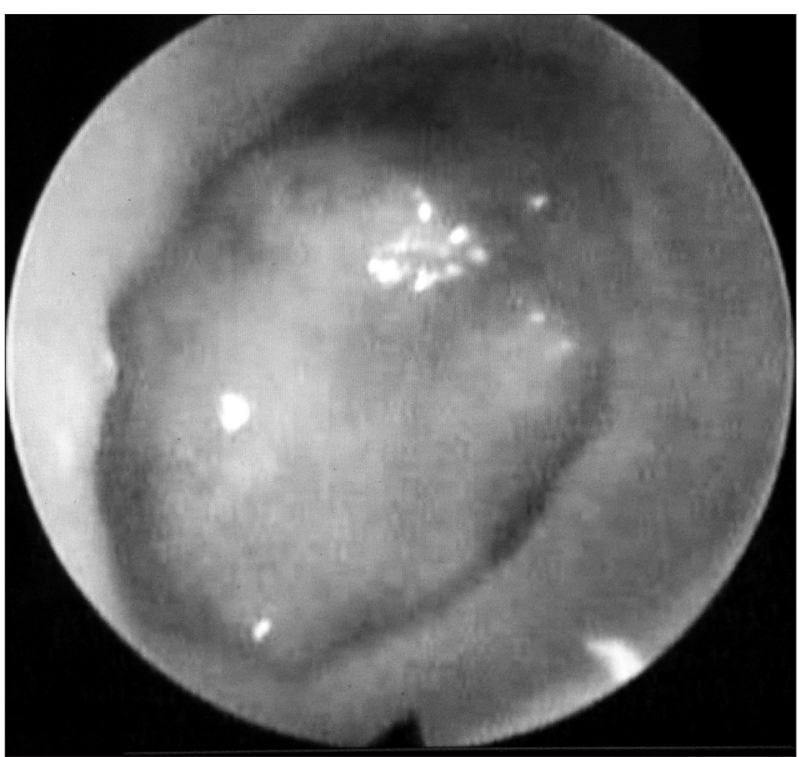

Figure 3. Right middle ear with absent malleus-incus ossicles, thickened middle ear mucosa and patent Eustachian tube.

Table 1. Pure tone audiometry without and with the "cotton wick"

\begin{tabular}{|c|c|c|c|c|c|c|c|c|}
\hline & \multicolumn{4}{|c|}{ No "cotton wick" } & \multicolumn{4}{|c|}{ With "cotton wick" } \\
\hline & $500 \mathrm{~Hz}$ & $1 \mathrm{kHz}$ & $2 \mathrm{KHz}$ & $4 \mathrm{KHz}$ & $500 \mathrm{~Hz}$ & $1 \mathrm{kHz}$ & $2 \mathrm{KHz}$ & $4 \mathrm{KHz}$ \\
\hline $\begin{array}{l}\text { Air } \\
\text { conduction AD }\end{array}$ & 80 & 120 & 110 & 120 & 50 & 80 & 75 & 95 \\
\hline $\begin{array}{l}\text { Pure tone } \\
\text { average }\end{array}$ & 107.5 & & & & 75 & & & \\
\hline $\begin{array}{l}\text { Bone } \\
\text { conduction AD }\end{array}$ & 25 & 50 & 65 & NR at 65 & 25 & 50 & 65 & NR at 65 \\
\hline Air bone gap & 55 & 70 & 45 & 55 & 25 & 30 & 10 & 30 \\
\hline ABG gain & & & & & 30 & 40 & 35 & 25 \\
\hline$A B G$ average $d B$ & \multicolumn{4}{|l|}{56.25} & \multicolumn{4}{|l|}{23.75} \\
\hline
\end{tabular}




\section{LETTER TO THE EDITOR}

\section{DISCUSSION}

At different anatomic levels, mechanical sound energy is amplified and transmitted to the functional parts of the ear. The tympanic membrane and oval window ratio of 21:1 and malleus-incus lever mechanism ratio of 1.3:1 provide a $28 \mathrm{~dB}$ amplification of conductive hearing. ${ }^{1}$ This gain is reflected by frequency specific air-bone gaps which can range between $25-40 \mathrm{~dB}$. With the contribution from the external ear, the overall conductive gain is $60 \mathrm{~dB} .^{1,3}$ Damage to the auditory system often results in a loss of hearing sensitivity that is frequency - specific. The presence of a frequency - specific wide airbone gap suggests ossicular chain discontinuity among patients with chronic otitis media. ${ }^{4}$ Narrowing of the air-bone gap which in this case was provided by insertion of the cotton wick may lead to at least partial restoration of ossicular coupling.

The ability of the cotton wick to improve hearing may be attributed to its possession of characteristics for sound conduction and acoustic impedance such as stiffness, resistance and mass. The effectiveness of the cotton wick was reported to be dependent on its positioning in the ear; the patient would have to insert the wick down to the level of the promontory or oval window occasionally blow his nose or reposition the cotton wick to achieve an acceptable hearing level. However, for a patient with completely deaf contralateral ear, a $32.5 \mathrm{~dB}$ gain in hearing is very pronounced and significant.

The hearing gain produced by the cotton wick only amplified the air conductive component of hearing but not bone conduction. While it afforded amplification of sound and a route of medicine administration, it may also have contributed to sensorineural hearing loss brought about by ototoxicity of medications and thickening of the oval and round window from chronic irritation. For this reason, utmost caution must be advised before considering use of a "cotton wick" to amplify hearing in this manner-- a practice we do not endorse.

The cotton wick may have served as a vibrating piston on top of the oval window which amplified hearing. Such a mechanism may conceivably prognosticate potential gain from a contemplated tympanoplasty in the same way that the "paper patch test" ${ }^{15}$ predicts simple myringoplasty outcomes. Having said that, the diagnostic utility of such a cotton wick requires further investigation before potential clinical applications such as prognostication of tympanoplasty are theorized. Could future studies show that a preoperative cotton wick (or equivalent device) may approximate potential gains from a good tympanoplasty with ossiculoplasty in a patient with total tympanic perforation and ossicular chain loss?

Sincerely,

Ryner Jose D. Carrillo, MD, MSc

Precious Eunice R. Grullo, MD, MPH

Maria Luz M. San Agustin, RN, MClinAudio

1. Chien W, Lee D. Physiology of the auditory system. In: Flint P, Haughey B, Lund V, Niparko J, Robbins $T$, Thomas $R$, Lesperance $M$, editors. Cummings otolaryngology head and neck surgery. $6^{\text {th }}$ ed. Canada: Saunders; 2015. p1995.

2. Hearing aid museum.com [homepage in the internet]. Philadelphia: Center for Hearing; c20062015. [cited 2015 Aug 10]. Available from: http://www.hearingaidmuseum.com/gallery.htm

3. Schlauch R, Nelson P. Basic test and procedures. In: Katz J editor. Handbook of clinical audiology, $7^{\text {th }}$ ed. Philadelphia: Wolters Kluwer Health; 2015. p30.

4. Carrillo RJC, Yang NW, Abes GT. Relationship of Pure Tone Audiometry and Ossicular Discontinuity in Chronic Suppurative Otitis Media. Philipp J Otolaryngol Head Neck Surg. 2006 21(1-2):5-10.

5. Triana R. Revision tympanoplasty. In: Carrasco V and Pillsbury H. Revision otologic surgery. New York: Thieme Medical Publishing; 1997. p.81-82.
} 